\title{
SOLVABILITY OF FACTORIZED FINITE GROUPS
}

\author{
ANGEL CAROCCA \\ Facultad de Matemáticas, Pontificia Universidad Católica de Chile, Casilla 306, Santiago 22, Chile \\ e-mail:acarocca@mat.puc.cl
}

(Received 9 October, 1998)

\begin{abstract}
Using classification theorems of simple groups, we give a proof of a conjecture on factorized finite groups which is an extension of a well known theorem due to P. Hall.
\end{abstract}

1991 Mathematics Subject Classification. Primary 20D10, 20D40; secondary $20 \mathrm{~F} 16$.

Let $G$ be a finite group and $G=G_{1} G_{2}$, where $G_{1}$ and $G_{2}$ are subgroups of $G$. There are a number of results in which one can deduce the solvability of $G$ from suitable conditions on $G_{1}$ and $G_{2}$ (see for instance [1]). According to a famous result of P. Hall ([5] and [6]), a finite group $G$ is solvable if and only if $G=P_{1} P_{2} \ldots P_{m}$ where $P_{i} \in S y l_{p_{i}} G$ and $P_{i} P_{j}=P_{j} P_{i}$ for all $i, j \in\{1, \ldots, m\}$.

Using classification theorems of simple groups, in this note we present an extension of the cited theorem of Hall. For this, we consider the following definition.

Let $\mathcal{S}$ be the class of all solvable groups. Two subgroups $G_{1}, G_{2}$ of a given group $G$ are $\mathcal{S}$-connected whenever for each $x \in G_{1}, y \in G_{2}$ we have $<x, y>\in \mathcal{S}$.

Considering this definition we prove the following theorem, which proves the conjecture formulated in [2].

THeOREM. Let $G=G_{1} G_{2} \ldots . G_{m}$ be a group such that $G_{1}, \ldots ., G_{m}$ are solvable subgroups of $G$. If $G_{1}, \ldots, G_{m}$ are pairwise permutable and pairwise $\mathcal{S}$-connected, then $G$ is solvable.

2. Preliminary results. In this section, we collect some of the results that are needed. If $G$ is the product of two solvable subgroups, it is known that $G$ is not necessarily solvable. Particular cases of finite groups factorizable by two subgroups were studied by many authors. Kazarin [8] studied the general case and obtained the following result.

2.1. Lemma (Kazarin [8].) Let $G=G_{1} G_{2}$ be a group with $G_{1}$ and $G_{2}$ solvable subgroups of $G$. If all composition factors of $G$ are known groups, then the nonabelian simple composition factors of $G$ belong to the following list of groups:

(a) $\operatorname{PSL}(2, q)$ with $q>3$,

(b) $\mathbf{M}_{11}$,

*This research was partially supported by FONDECYT 8970007 and Cátedra Presidencial en Geometríca. 
(c) $\operatorname{PSL}(3, q)$ with $q<9$,

(d) $\operatorname{PSp}(4,3)$,

(e) $\operatorname{PSU}(3,8)$,

(f) $\operatorname{PSL}(4,2)$.

A consequence of Kazarin's result is the following lemma.

2.2. Lemma (Fisman [3].) Let $G=G_{1} G_{2} \ldots G_{m}$ be a group such that $G_{i} G_{j}$ is a solvable subgroup, for every $i, j \in\{1,2, \ldots, m\}$. Then $G$ is solvable.

REMARK 1 . Let $G=\operatorname{PSL}(2, q)$ with $q=p^{l}$ and $p$ a prime number. The following properties of $G$ are well known.

(a) $|G|=\frac{q(q+1)(q-1)}{d}$ where $d=(2, q-1)$.

(b) A Sylow- $p$-subgroup $P$ of $G$ is elementary abelian of order $q=p^{l}$ and $P$ is disjoint from its conjugates. Further $\left|G: \mathbf{N}_{G}(P)\right|=q+1$.

(c) If $r$ is a prime distinct from $p$ or 2, then a Sylow- $r$-subgroup of $G$ is cyclic.

(d) If $p$ is odd, then a Sylow-2-subgroup of $G$ is dihedral.

(e) $G$ contains cyclic subgroups $U$ of orders $s=\frac{q+1}{d}$ and $s=\frac{q-1}{d}$. For each $1 \neq u \in U$, we have that $\mathbf{N}_{G}(<u>)$ is a dihedral group of order $2 s$.

For a proof see [7, Satz 8.2/8.3/8.4, p.192].

2.3. Lemma Let $G=G_{1} G_{2}=G_{1} N=G_{2} N$ be a group, where $G_{1}$ and $G_{2}$ are solvable subgroups of $G$ and $N$ is the unique minimal normal subgroup of $G$ and $N$ is nonsolvable. Then

(a) $G_{1}$ acts transitively as a permutation group on the set of normal subgroups of $N$ and $G_{1} \cap N=\prod_{i}^{m} L_{i}$ for $N=\prod_{i}^{m} N_{i}$ (with $N_{i} \cong N_{j}$ ) for every $i, j \in\{1, \ldots, m\}$ and
$L_{i}=N_{i} \cap G_{1}$.

(b) $\left|N_{1}\right|$ divides $\left|\operatorname{Out}\left(N_{1}\right)\right|\left|N_{1} \cap G_{1}\right|\left|N_{1} \cap G_{2}\right|$.

For a proof see [8, Lemmas 2.3 and 2.5].

2.4. Lemma Let $G=<x><y>$ be a group. Then $G$ is supersolvable. In particular, the Sylow-p-subgroup of $G$, where $p$ is the largest prime divisor of $|G|$, is normal in $G$.

For a proof see [7, Satz 10.1, p.722].

Remark 2. Let $G$ be a solvable group, $P \in S y l_{p}(G)$ and $Q \in S y l_{q}(G)$. If $\mathbf{N}_{G}(P)=P$ and $\mathbf{N}_{G}(Q)=Q$, then $p=q$.

This is a corollary of Carter's Theorem [7, Satz 12.2, p.736].

3. Proof of the theorem. Suppose that the Theorem 1 is false and let $G$ be a counterexample of smallest order with $m$ least possible. By Lemma 2.2, we have that 
$G=G_{1} G_{2}$. Clearly the hypothesis is inherited by factor groups. Hence $G$ has a unique minimal normal subgroup $N$, and $N$ is nonsolvable. Since $G_{1} N=G_{1}\left(G_{1} N \cap G_{2}\right)$ and $G_{1}, G_{1} N \cap G_{2}$ are $\mathcal{S}$-connected solvable subgroups of $G_{1} N$, we have that $G_{1} N=G=G_{2} N$, by the minimality of $G$.

By Lemma 2.1, we have that the composition factors of $G$ belong to the following list:

(a) $\operatorname{PSL}(2, q)$ with $q>3$,

(b) $\mathbf{M}_{11}$,

(c) $\operatorname{PSL}(3, q)$ with $q<9$,

(d) $\operatorname{PSp}(4,3)$,

(e) $\operatorname{PSU}(3,8)$,

(f) $\operatorname{PSL}(4,2)$.

From now on we denote by $L$ a nonabelian simple composition factor of $G$. By the above arguments $L \leq G$. Put $H=L \cap G_{1}$ and $K=L \cap G_{2}$.

(I) Assume that $L \cong \mathbf{P S L}(2, q)$ with $q=p^{a}$ an odd number.

Let $r \in \pi(L)-\{2, p\}$ and $R$ be an $r$-subgroup of $L$. Since $\left(\frac{q+1}{2}, \frac{q-1}{2}\right)=1$ we have that $\left|\mathbf{N}_{L}(R)\right|=2 s$ with $s=\frac{q+1}{2}$ or $s=\frac{q-1}{2}$, by Remark 1 (e). In particular $p$ is not a divisor of $\left|\mathbf{N}_{L}(R)\right|$.

Let $x$ be a $p$-element of $H$ and $y$ an $r$-element of $H$ or $K$. Since $M=<x, y>$ is solvable there exist $P_{1} \in S y l_{p}(M)$ and $R_{1} \in S y l_{r}(M)$ such that $P_{1} R_{1}=R_{1} P_{1}$. Since $p$ is not a divisor of $\left|\mathbf{N}_{L}\left(R_{1}\right)\right|$, by Remark 2 we have that $r$ divides $\left|\mathbf{N}_{M}\left(P_{1}\right)\right|$. Hence, if $P \in \operatorname{Syl}_{p}(L)$, then $r$ divides $\left|\mathbf{N}_{L}(P)\right|$ by Remark 1 (a). It follows that every odd prime number in $\pi(L)$ divides $\left|\mathbf{N}_{L}(P)\right|=\frac{q(q-1)}{2}$, by Lemma 2.3 (b). Again, since $\left(\frac{q+1}{2}, \frac{q-1}{2}\right)=1$ we have $q+1=2^{s}$. We shall obtain a contradiction proving that 2 divides $\left|\mathbf{N}_{L}(P)\right|$.

Let $w$ be a 2-element of $H$ or $K$ and $M=<x, w>$. Let $S_{1} \in S y l_{2}(M)$ and $P_{1} \in S y l_{p}(M)$ be such that $P_{1} S_{1}=S_{1} P_{1}$. Since 2 does not divide $\left|\mathbf{N}_{L}(P)\right|$ it follows that 2 does not divide $\left|\mathbf{N}_{M}\left(P_{1}\right)\right|$ by Remark 1 (a). Hence $p$ divides $\left|\mathbf{N}_{M}\left(S_{1}\right)\right|$. Let $P_{2} \in \operatorname{Syl}_{p}\left(\mathbf{N}_{M}\left(S_{1}\right)\right)$. If there exist a subgroup $Z$ of $S_{1}$ of order two normalized by some subgroup $P_{3}$ of $P_{2}$, then $P_{3} \unlhd Z P_{3}$ and we obtain a contradiction. Therefore, since $S_{1}$ is cyclic or dihedral, we have that $S_{1}$ is of order 4 and $P_{2}$ acts faithfully on $S_{1}$. Hence $P_{2} \leq \mathbf{G L}(2,2)$ and $p=3$. It follows that $q=3$, a contradiction.

(II) Assume that $L \cong \operatorname{PSL}\left(2,2^{n}\right)$.

Let $p$ be the largest prime and $r \neq p$ an odd prime both dividing $\omega|L|, x$ be a $p$-element of $H$ and $y$ an $r$-element of $H$ or $K$. Since $M=<x, y>$ is solvable, there are $P_{1}=<x_{1}>\in S y l_{p}(M)$ and $R_{1}=<y_{1}>\in S y l_{r}\left(G_{2}\right)$ such that $P_{1} R_{1}=R_{1} P_{1}$. Hence, by Lemma 2.4 it follows that $P_{1} \unlhd R_{1} P_{1}$. We deduce that every odd prime in $\pi(L)$ divides $\omega$ (by Remark $1(\mathrm{e})$ ) and $2^{n}=2$, a contradiction.

(III) Assume that $L$ is isomorphic to some group of the following list: $\{\operatorname{PSL}(3, q)$ with $q<9$ (here $q \neq 2$ since $\mathbf{P S L}(3,2) \cong \mathbf{P S L}(2,7)), \mathbf{M}_{11}, \mathbf{P S p}(4,3), \mathbf{P S L}(4,2)$ or $\operatorname{PSU}(3,8)\}$.

By Lemma 2.3 (b) we have that $|L|$ divides $|\operatorname{Out}(L)||H||K|$. Therefore, for every $\{p, q\} \subseteq \pi(L)$ there is a solvable $\{p, q\}$-subgroup $S$ of $L$. Since 
PSL $(3,3)$ does not have a $\{2,13\}$-subgroup, $\operatorname{PSL}(3,4)$ does not have a $\{5,7\}$-subgroup,

PSL $(3,5)$ does not have a $\{5,31\}$-subgroup, PSL $(3,7)$ does not have a $\{7,19\}$-subgroup, PSL $(3,8)$ does not have a $\{7,73\}$-subgroup, $\mathbf{M}_{11}$ does not have a $\{3,11\}$-subgroup, $\operatorname{PSp}(4,3)$ does not have a $\{3,5\}$-subgroup, PSU $(3,8)$ does not have a $\{7,19\}$-subgroup, $\operatorname{PSL}(4,2)$ does not have a $\{5,7\}$-subgroup,

we have a contradiction.

\section{REFERENCES}

1. B. Amberg, S. Franciosi and F. de Giovanni, Products of groups (Clarendon Press, Oxford, 1992).

2. A. Carocca, A note on the product of $\mathcal{F}$-subgroups in a finite group, Proc. Edinburgh Math. Soc. (2) 39 (1996), 37-42.

3. E. Fisman, Product of solvable subgroups of a finite group, Arch. Math. (Basel) 61 (1993), 201-205.

4. D. Gorenstein, Finite groups. (New York, Harper and Row, 1968).

5. P. Hall, A note on soluble groups, J. London Math. Soc. 3 (1928), 98-105. $188-200$

6. P. Hall, A characteristic property of soluble groups, J. London Math. Soc. 12 (1938),

7. B. Huppert, Endliche Gruppen I (Springer-Verlag, 1967).

8. L. Kazarin, Product of two solvable subgroups, Comm. Algebra 14 (1986), 1001-1066. 